\title{
NUMERICAL HOMOGENIZATION OF WELL SINGULARITIES IN THE FLOW TRANSPORT THROUGH HETEROGENEOUS POROUS MEDIA: FULLY DISCRETE SCHEME
}

\author{
MEIQUn JiAnG ${ }^{1}$ AND XingYe YuE ${ }^{2}$
}

\begin{abstract}
Motivated by well-driven flow transport in porous media, Chen and Yue proposed a numerical homogenization method for Green function [Multiscale Model. Simul. 1 (2003) 260-303]. In that paper, the authors focused on the well pore pressure, so the local error analysis in maximum norm was presented. As a continuation, we will consider a fully discrete scheme and its multiscale error analysis on the velocity field.
\end{abstract}

Mathematics Subject Classification. 65N30, 65N15.

Received June 15, 2006. Revised March 7, 2007.

\section{INTRODUCTION}

Upscaling or homogenization methods have become the powerful tools for the macroscopic modeling of the flow transport in the heterogenous porous media $[1,4,6,14,16]$. It was known that for the well-driven flow in the porous media, the standard upscaling methods did not work in the vicinity of the wells $[5,13]$. In the recent paper [3], Chen and Yue developed a multiscale coarse grid algorithm for solving steady flow problem involving well singularities in heterogeneous porous medium based on the over-sampling multiscale finite element method (MsFEM) [10]. The remedy was that the well singularities (Dirac sources) of the problem were first resolved locally and then were removed; the left part could be formulated in a variational form and was solved by the multiscale finite element method.

In the previous work, focusing on the well bore pressure, Chen and Yue presented the local error analysis in maximum norm. Though the well bore pressure plays a key role in the well control [15], the flow pattern in the whole reservoir is also very important in the engineering. In this paper, we are going to consider the multiscale error analysis for the flow velocity field, i.e. in the energy norm.

The general idea of MsFEM is to construct finite element basis functions that capture the small scale information of the leading order differential operator. In practical implementation, this requires numerically solving a series of differential equations associated with the differential operator. It seems that the fully discrete error is not considered in the analysis on MsFEM so far $(c f .[2,3,7,11])$, except for in $[8,9]$, a numerical homogenization method related to MsFEM was proposed and the fully discrete error analysis was presented for monotone elliptic operators and quasi-convex energies. However in these two works, only the error between the numerical

Keywords and phrases. Numerical homogenization, well-driven flow, heterogeneous porous medium, multiscale finite element.

1 Department of Mathematics, Suzhou University, Suzhou 215006, China. mqjiang@suda.edu.cn

2 Department of Mathematics, University of Science and Technology of China, Hefei 230026, China. xyyue@ustc.edu.cn

(c) EDP Sciences, SMAI 2007 
solution and the solution of the homogenized equation was considered. In present work, we will analyze the error between the fully discrete solution of over-sampling MsFEM and the exact solution of the original problem.

Although the main singularity induced by the Dirac source is removed, what's left is only a piecewise $H^{2}$ function. In fact, the right hand side of the governing equation for the left part does not belong to $L^{2}$ (see (1.3) below). The lack of global regularity also brings some difficulties in the error analysis.

We now recall the problem and one of the main ideas in the previous study [3]. Let $\Omega \subset \mathbf{R}^{2}$ a bounded domain with Lipschitz boundary $\Gamma$. We consider the following problem

$$
\begin{aligned}
-\operatorname{div}\left(K_{\varepsilon}(x) \nabla u_{\varepsilon}\right) & =\delta_{x_{0}}, & & \text { in } \Omega, \\
u_{\varepsilon} & =0, & & \text { on } \Gamma,
\end{aligned}
$$

where $0<\varepsilon \ll 1$ is the ratio between the characteristic length scale of the micro structure and the macroscopic scale of the porous media, and $K_{\varepsilon}=K\left(x, \frac{x}{\varepsilon}\right)$.

Let $\Omega_{0}$ be a small sub-domain inside $\Omega$ such that $x_{0} \in \Omega_{0}, H_{0}=\operatorname{dist}\left(x_{0}, \partial \Omega_{0}\right)$ and there exists a constant $C>0$ satisfying that $\operatorname{diam}\left(\Omega_{0}\right) \leq C H_{0}$. Let $G_{\varepsilon}$ be the local Green function associated with the domain $\Omega_{0}$

$$
-\operatorname{div}\left(K_{\varepsilon}(x) \nabla G_{\varepsilon}\right)=\delta_{x_{0}} \quad \text { in } \Omega_{0},\left.\quad G_{\varepsilon}\right|_{\Sigma_{0}}=0,
$$

where $\Sigma_{0}=\partial \Omega_{0}$. Let $G_{\varepsilon}=0$ for $x \in \Omega \backslash \bar{\Omega}_{0}$ and set $\zeta_{\varepsilon}=u_{\varepsilon}-G_{\varepsilon}$, then $\zeta_{\varepsilon} \in H_{0}^{1}(\Omega)$ satisfies the following variational form

$$
\int_{\Omega} K_{\varepsilon}(x) \nabla \zeta_{\varepsilon} \nabla v \mathrm{~d} x=-\int_{\Sigma_{0}} K_{\varepsilon} \frac{\partial G_{\varepsilon}}{\partial \nu} v \mathrm{~d} x \quad \forall v \in C_{0}^{\infty}(\Omega)
$$

The main singularity of the original solution $u_{\varepsilon}$ is removed and the over-sampling MsFEM [10] can be used to discretize the above variational form on a coarse grid. The local convergence in maximum norm of the method was established in [3] for locally periodic coefficients, i.e. assuming $K_{\varepsilon}(x)=K(x, x / \varepsilon)$, where $K(x, \cdot)$ is periodic with respect to the unit square $Y$.

The paper is organized as follows: in Section 2 we introduce the fully discrete multiscale algorithm based on the weak formulation (1.3). In Section 3 we list some results on the homogenization of Green function and establish some new homogenization results for $\zeta_{\varepsilon}$, which will be used in Section 4 to complete the multiscale error analysis for the velocity field. In the whole paper, $C>0$ is a general constant independent of the parameters $\varepsilon, H_{0}$, the fine grid scale $h$ and the coarse grid scale $H$.

\section{The FUlLY DISCRETE SCHEME}

In this section we are going to recall the multiscale method to solve the problem (1.3).

Let $\mathcal{M}_{H}$ be a regular and quasi-uniform triangulation of $\Omega$ and $V_{H}$ the standard conforming linear finite element space over $\mathcal{M}_{H}$. For any $T \in \mathcal{M}_{H}$ with nodes $\left\{x_{i}^{T}\right\}_{i=1}^{3}$, let $H_{T}$ denote the size of $T, P_{1}(T)$ the set of linear polynomials defined in $T$, and $\left\{\varphi_{i}^{T}\right\}_{i=1}^{3}$ the basis of $P_{1}(T)$ satisfying $\varphi_{i}^{T}\left(x_{j}^{T}\right)=\delta_{i j}, i, j=1,2,3$. For any $T \in \mathcal{M}_{H}$, we denote by $S=S(T)$ a macro-element which contains $T$ and satisfies the following condition.

(H1) $H_{S} \leq C_{1} H_{T}$ and $\operatorname{dist}(\partial T, \partial S) \geq \delta_{0} H_{T}$ for some positive constants $C_{1}, \delta_{0}$ independent of $H$. The minimum angles of $S(T)$ is bounded below by some positive constant $\theta_{0}$ independent of $H$.

Let $M S(S)$ be the multiscale finite element space spanned by $\psi_{i}^{S}, i=1,2,3$, with $\psi_{i}^{S} \in H^{1}(S)$ being the solution of the problem

$$
-\operatorname{div}\left(K_{\varepsilon}(x) \nabla \psi_{i}^{S}\right)=0 \quad \text { in } S,\left.\quad \psi_{i}^{S}\right|_{\partial S}=\varphi_{i}^{S}
$$


Here $\left\{\varphi_{i}^{S}\right\}_{i=1}^{3}$ is the nodal basis of $P_{1}(S)$ such that $\varphi_{i}^{S}\left(x_{j}^{S}\right)=\delta_{i j}, i, j=1,2,3$. Then we define the over-sampling finite element basis over $T$ by

$$
\bar{\psi}_{i}^{T}=\left.c_{i j}^{T} \psi_{i}^{S}\right|_{T} \quad \text { in } T,
$$

with the constants $c_{i j}^{T}$ so chosen that $\varphi_{i}^{T}=\left.c_{i j}^{T} \varphi_{j}^{S}\right|_{T}$ in $T$.

Let $O M S(T)=\operatorname{span}\left\{\bar{\psi}_{i}^{T}\right\}_{i=1}^{3}$ and $\Pi_{T}: O M S(T) \rightarrow P_{1}(T)$ the projection

$$
\Pi_{T} \psi=c_{i} \varphi_{i}^{T} \quad \text { if } \psi=c_{i} \bar{\psi}_{i}^{T} \in O M S(T) .
$$

Let $\bar{X}_{H}$ be the finite element space $\bar{X}_{H}=\left\{\psi_{H}:\left.\psi_{H}\right|_{T} \in O M S(T), \forall T \in \mathcal{M}_{H}\right\}$ and define $\Pi_{H}: \bar{X}_{H} \rightarrow$ $\Pi_{T \in \mathcal{M}_{H}} P_{1}(T)$ through the relation $\left.\Pi_{H} \psi_{H}\right|_{T}=\Pi_{T} \psi_{H}$ for any $T \in \mathcal{M}_{H}, \psi_{H} \in \bar{X}_{H}$. The over-sampling finite element space is then defined as

$$
X_{H}=\left\{\psi_{H} \in \bar{X}_{H}: \Pi_{H} \psi_{H} \in V_{H} \subset H^{1}(\Omega)\right\} .
$$

Taking the boundary condition into account, we set $X_{H}^{0}=\left\{\psi_{H} \in X_{H}: \Pi_{H} \psi_{H}=0\right.$ on $\left.\Gamma\right\}$.

The fully discrete counterparts of $X_{H}$ and $X_{H}^{0}$ are denoted by $Y_{H}$ and $Y_{H}^{0}$, which can be defined in the same way as above, except that the element bases $\psi_{i}^{S}, i=1,2,3$ in (2.1) are replaced by their conforming piecewise linear finite element approximation $\psi_{i}^{h}, i=1,2,3$ on a fine grid of size $h$ resolving the small scale $\varepsilon$ over the macro element $S$.

Note that we have now three types of finite element spaces over the same triangulation $\mathcal{M}_{H}$ :

$$
\begin{aligned}
& V_{H} \text { - the conforming piecewise linear finite element space; } \\
& X_{H} \text { - the common multiscale finite element space; } \\
& Y_{H} \text { - the multiscale finite element space with fully discrete basis functions. }
\end{aligned}
$$

In numerical implementation, not only should the multiscale finite element basis functions be numerically constructed, but also the local Green function $G_{\varepsilon}$ in $\Omega_{0}$ in (1.3). We are going to approximate it by its conforming piecewise linear finite element solution $G_{h}$ on a fine grid with size $h$ resolving the scale $\varepsilon$ over $\Omega_{0}$. In order to avoid to approximate the normal derivative $\frac{\partial G_{\varepsilon}}{\partial \nu}$ on the subdomain boundary $\Sigma_{0}=\partial \Omega_{0}$, we need the following equivalent form of $(1.3)$

$$
\int_{\Omega} K_{\varepsilon}(x) \nabla \zeta_{\varepsilon} \nabla v \mathrm{~d} x=\int_{\Omega_{0}} K_{\varepsilon} \nabla G_{\varepsilon} \nabla(\phi v) \mathrm{d} x \quad \forall v \in C_{0}^{\infty}(\Omega),
$$

where $\phi$ is a cut-off function such that $\phi \in C^{1}\left(\Omega_{0}\right), \phi \equiv 1$ in $\Omega_{0} \backslash B\left(x_{0}, 3 H_{0} / 4\right), \phi \equiv 0$ in $B\left(x_{0}, H_{0} / 2\right)$. Here $B(x, r) \subset \mathbf{R}^{2}$ denotes a ball centered at $x$ with a radius of $r$.

Now we define the fully discrete scheme: Find $\zeta_{H} \in Y_{H}^{0}$ such that

$$
\sum_{T \in \mathcal{M}_{H}} \int_{T} K_{\varepsilon}(x) \nabla \zeta_{H} \nabla \chi_{H} \mathrm{~d} x=-\int_{\Omega_{0}} K_{\varepsilon} \nabla G_{h} \nabla\left(\phi \hat{\chi}_{H}\right) \mathrm{d} x \quad \forall \chi_{H} \in Y_{H}^{0},
$$

where $\hat{\chi}_{H}=\Pi_{H} \chi_{H} \in V_{H}^{0}=V_{H} \cap H_{0}^{1}(\Omega)$, the project $\Pi_{H}: Y_{H} \rightarrow V_{H}$ is defined in the same way as the one from $X_{H}$ to $V_{H}$ above.

If we make the following local periodicity assumption for the coefficient:

(H2) $K_{\varepsilon}(x)=K(x, x / \varepsilon)$ satisfies the uniform elliptic condition and $K \in C^{1}\left(\bar{\Omega} ; C_{p}^{1}\left(\mathbf{R}^{2}\right)\right)$, where $C_{p}^{1}\left(\mathbf{R}^{2}\right)$ stands for the collection of all $C^{1}\left(\mathbf{R}^{2}\right)$ periodic functions with respect to the unit square $Y$, then for the fully discrete scheme, we have: 
Theorem 2.1. Let the assumptions (H1)-(H2) be fulfilled. Then there exists a constant $C$ independent of $\varepsilon, h$, $H$ and $H_{0}$ such that for $0<h \ll \varepsilon \ll H \ll 1$, the following error estimate is valid

$$
\begin{aligned}
\left\|\zeta_{\varepsilon}-\zeta_{H}\right\|_{H} \leq & C\left(\left(H+\frac{\varepsilon}{H}+\frac{\varepsilon}{H_{0}}+\frac{H}{H_{0}}+\frac{h}{\varepsilon}\right)\left|\ln H_{0}\right|+\frac{\varepsilon}{H_{0}}|\ln \varepsilon|\right. \\
& \left.+\frac{\varepsilon}{H_{0} H}+\sqrt{\frac{\varepsilon}{H_{0}}}+\frac{h}{\varepsilon H_{0}}|\ln H|^{1 / 2}\right),
\end{aligned}
$$

where $\left\|\chi_{H}\right\|_{H}=\left\|\chi_{H}\right\|_{H, \Omega}$ and $\left\|\chi_{H}\right\|_{H, D}=\left(\sum_{T \in \mathcal{M}_{H} \cap D} \int_{T}\left|\nabla \chi_{H}\right|^{2} \mathrm{~d} x\right)^{1 / 2}$.

Remark. Here we restrict ourself to a two dimensional Green function problem. In fact, in oil industry both the vertical and horizontal well should be regarded as line sources. In the discrete sense at each cross-section, we have to treat a two dimensional Dirac source. That's the reason why most of the well treatment problem in engineering is two dimensional. However most of our results and the analysis here are valid for three dimensional problem, except for the homogenization results and some a priori estimates related to two dimensional Green function.

\section{Homogenization Results}

In this section we list several results of standard homogenization theory, and prove some new homogenization results for $\zeta_{\varepsilon}$, which will play a basic role in the subsequent analysis.

Let $D \subseteq \Omega$ be a bounded domain with Lipschitz boundary. Given $f \in L^{2}(D)$, we consider the following problem with

$$
\int_{D} a\left(x, \frac{x}{\varepsilon}\right) \nabla w_{\varepsilon} \nabla \varphi \mathrm{d} x=\int_{D} f \varphi \mathrm{d} x \quad \forall \varphi \in H_{0}^{1}(D) .
$$

Here $a(x, x / \varepsilon)=\left(a_{i j}(x, x / \varepsilon)\right)$ is a symmetric matrix which satisfies the uniform ellipticity condition (in our application $\left.a(x, x / \varepsilon)=K_{\varepsilon}(x) \mathbf{I}\right)$. Furthermore, we assume that $a_{i j} \in C^{1}\left(\bar{D} ; C_{p}^{1}\left(\mathbf{R}^{2}\right)\right)$.

Let $w_{0} \in H_{0}^{1}(D)$ be the unique solution of the homogenized problem

$$
\int_{D} a^{*}(x) \nabla w_{0} \nabla \varphi \mathrm{d} x=\int_{D} f \varphi \mathrm{d} x \quad \forall \varphi \in H_{0}^{1}(D),
$$

where $a^{*}(x)=\left(a_{i j}^{*}(x)\right)$ with

$$
a_{i j}^{*}(x)=\frac{1}{|Y|} \int_{Y} a_{i k}(x, y)\left(\delta_{k j}-\frac{\partial \chi^{j}}{\partial y_{k}}(x, y)\right) \mathrm{d} y,
$$

and $\chi^{j}(x, y)$ is the periodic solution of the cell problem

$$
\frac{\partial}{\partial y_{i}}\left(a_{i k}(x, y) \frac{\partial \chi^{j}}{\partial y_{k}}(x, y)\right)=\frac{\partial}{\partial y_{i}} a_{i j}(x, y) \quad \text { in } Y, \quad \int_{Y} \chi^{j}(x, y) \mathrm{d} y=0 .
$$

Here $\delta_{k j}$ is the Kronecker delta, i.e. $\delta_{k j}=1$ for $k=j$, and $\delta_{k j}=0$ for $k \neq j$.

Set $w_{1}^{\varepsilon}(x)=w_{0}(x)-\varepsilon \chi^{k}\left(x, \frac{x}{\varepsilon}\right) \frac{\partial w_{0}}{\partial x_{k}}$. Let $\theta_{\varepsilon}=\theta_{\varepsilon}\left(w_{0}\right) \in H^{1}(D)$ be the boundary corrector that satisfies

$$
-\operatorname{div}\left(a\left(x, \frac{x}{\varepsilon}\right) \nabla \theta_{\varepsilon}\right)=0 \quad \text { in } D,\left.\quad \theta_{\varepsilon}\right|_{\partial D}=\chi^{k}\left(x, \frac{x}{\varepsilon}\right) \frac{\partial w_{0}}{\partial x_{k}} .
$$

The following theorem is known (cf. e.g. $[2,12])$. 
Theorem 3.1. Assume that $w_{0} \in H^{2}(D) \cap W^{1, \infty}(D)$. Then there exists a constant $C$ independent of $\varepsilon$, the domain $D$, and the function $f$ such that

$$
\left\|\nabla w_{\varepsilon}-\nabla\left(w_{1}^{\varepsilon}+\varepsilon \theta_{\varepsilon}\right)\right\|_{0, D} \leq C \varepsilon\left(\left|w_{0}\right|_{2, D}+\left|w_{0}\right|_{1, D}\right),
$$

and for the boundary corrector $\theta_{\varepsilon}$, there exists the following estimate

$$
\left\|\varepsilon \nabla \theta_{\varepsilon}\right\|_{0, D} \leq C \varepsilon\left(\left|w_{0}\right|_{2, D}+\left|w_{0}\right|_{1, D}\right)+C \sqrt{\varepsilon|\partial D|}\left|w_{0}\right|_{1, \infty, D},
$$

where $|\partial D|$ stands for the length of the boundary $\partial D$.

Furthermore, after checking the proof of Theorem 3.1 ( $c f .[2])$, we may obtain a more precise result for the boundary corrector

$$
\left\|\varepsilon \nabla \theta_{\varepsilon}\right\|_{0, D} \leq C \varepsilon\left(\left|w_{0}\right|_{2, D_{\varepsilon}}+\left|w_{0}\right|_{1, D_{\varepsilon}}\right)+C \sqrt{\varepsilon|\partial D|}\left|w_{0}\right|_{1, \infty, D_{\varepsilon}},
$$

where $D_{\varepsilon}=\{x \in D$ : dist $(x, \partial D) \leq \varepsilon\} \subset D$ is a $\varepsilon$-neighborhood of the boundary $\partial D$. This is true because one can choose the cut-off function vanishing outside the subset $D_{\varepsilon}$ in the proof of (3.7).

Now we are going to establish the homogenization results for $\zeta_{\varepsilon}$. As known, in standard homogenization theory, the asymptotic expansion (e.g. (3.6)) is valid under the assumption that the source terms be in $L^{2}$. Note that for the problem (1.3), this assumption is not true. Though the main singularity has been removed, we cannot expect that $\zeta_{\varepsilon} \in H^{2}(\Omega)$. However, benefiting from the special structure of $\zeta_{\varepsilon}$, we can deduce a piecewise asymptotic expansion for it.

We first define the following homogenized problems for $u_{0}, G_{0}$ and $\zeta_{0}$ satisfying

$$
\begin{array}{ll}
\left(K^{*} \nabla u_{0}, \nabla v\right)=v\left(x_{0}\right), & \forall v \in H_{0}^{1}(\Omega) \cap C(\Omega), \\
\left(K^{*} \nabla G_{0}, \nabla v\right)=v\left(x_{0}\right), & \forall v \in H_{0}^{1}\left(\Omega_{0}\right) \cap C\left(\Omega_{0}\right)
\end{array}
$$

and

$$
\int_{\Omega} K^{*} \nabla \zeta_{0} \nabla \chi \mathrm{d} x=-\int_{\partial \Omega_{0}} K^{*} \frac{\partial G_{0}}{\partial \nu} \chi, \quad \forall \chi \in H_{0}^{1}(\Omega)
$$

where $K^{*}$ is defined by (3.3).

Obviously,

$$
\zeta_{\varepsilon}=\left\{\begin{array}{cl}
u_{\varepsilon}, & x \in \Omega \backslash \Omega_{0} \\
u_{\varepsilon}-G_{\varepsilon}, & x \in \bar{\Omega}_{0},
\end{array} \zeta_{0}=\left\{\begin{array}{cl}
u_{0}, & x \in \Omega \backslash \Omega_{0} \\
u_{0}-G_{0}, & x \in \bar{\Omega}_{0}
\end{array}\right.\right.
$$

Then we present the main results of this section.

Theorem 3.2. Assume that $\operatorname{diam}\left(\Omega_{0}\right) \leq C H_{0}$, then there exists a constant $C$ independent of $\varepsilon$ and $H_{0}$, such that

$$
\begin{aligned}
& \left\|\nabla\left(\zeta_{\varepsilon}-\zeta_{0}+\varepsilon \chi_{i} \frac{\partial \zeta_{0}}{\partial x_{i}}-\varepsilon \theta_{\varepsilon}\left(\zeta_{0}\right)\right)\right\|_{0, \Omega \backslash \Omega_{0}} \leq C \frac{\varepsilon}{H_{0}}|\ln \varepsilon|, \\
& \left\|\nabla\left(\zeta_{\varepsilon}-\zeta_{0}+\varepsilon \chi_{i} \frac{\partial \zeta_{0}}{\partial x_{i}}-\varepsilon \bar{\theta}_{\varepsilon}\left(\zeta_{0}\right)\right)\right\|_{0, \Omega_{0}} \leq C\left(\frac{\varepsilon}{H_{0}}|\ln \varepsilon|+\sqrt{\frac{\varepsilon}{H_{0}}}\right),
\end{aligned}
$$

where $\theta_{\varepsilon}\left(\zeta_{0}\right)$ and $\bar{\theta}_{\varepsilon}\left(\zeta_{0}\right)$ are the boundary correctors for domains $\Omega$ and $\Omega_{0}$ respectively defined as (3.5). 
Before the proof, we list some useful results on $u_{\varepsilon}$ and $u_{0}$ in the previous work (cf. [3], Thm. 4.3, Thm. 4.5 and its proof and Lem. 4.4 respectively),

$$
\begin{aligned}
& \left\|u_{\varepsilon}-u_{0}\right\|_{0, \Omega \backslash B\left(x_{0}, r\right)} \leq C \varepsilon\left(1+\ln \frac{r}{\varepsilon}\right), \\
& \left\|\nabla\left(u_{\varepsilon}-u_{0}+\varepsilon \chi_{i} \frac{\partial u_{0}}{\partial x_{i}}-\varepsilon \theta_{\varepsilon}\left(u_{0}\right)\right)\right\|_{0, \Omega \backslash B\left(x_{0}, r\right)} \leq C \frac{\varepsilon}{r}|\ln \varepsilon|, \\
& \left|\nabla u_{0}(x)\right| \leq C r^{-1} \quad \text { for any } x \in \Omega \backslash B\left(x_{0}, r\right), \\
& \left\|D^{2} u_{0}\right\|_{0, \Omega \backslash B\left(x_{0}, r\right)} \leq C r^{-1} .
\end{aligned}
$$

Proof of Theorem 3.2. From (3.12), $\zeta_{\varepsilon}=u_{\varepsilon}, \zeta_{0}=u_{0}$ in $\Omega \backslash \Omega_{0}$. So (3.13) is just a local asymptotic expansion for the Green function $u_{\varepsilon}, c f$. (3.16).

To prove the second result (3.14), we first rewrite by (3.12),

$$
\begin{aligned}
& -\operatorname{div}\left(K_{\varepsilon} \nabla \zeta_{\varepsilon}\right)=0, \quad \text { in } \Omega_{0},\left.\quad \zeta_{\varepsilon}\right|_{\partial \Omega_{0}}=u_{\varepsilon}, \\
& -\operatorname{div}\left(K^{*} \nabla \zeta_{0}\right)=0, \quad \text { in } \Omega_{0},\left.\quad \zeta_{0}\right|_{\partial \Omega_{0}}=u_{0} .
\end{aligned}
$$

Define $\bar{\zeta}_{\varepsilon}$ by

$$
-\operatorname{div}\left(K_{\varepsilon} \nabla \bar{\zeta}_{\varepsilon}\right)=0, \quad \text { in } \Omega_{0},\left.\quad \bar{\zeta}_{\varepsilon}\right|_{\partial \Omega_{0}}=u_{\varepsilon}-u_{0},
$$

then $\zeta_{0}$ is the homogenization of $\left(\zeta_{\varepsilon}-\bar{\zeta}_{\varepsilon}\right)$. Therefore, by standard homogenization theory (3.1),

$$
\begin{aligned}
\left\|\nabla\left(\left(\zeta_{\varepsilon}-\bar{\zeta}_{\varepsilon}\right)-\zeta_{0}+\varepsilon \chi_{i} \frac{\partial \zeta_{0}}{\partial x_{i}}-\varepsilon \bar{\theta}_{\varepsilon}\left(\zeta_{0}\right)\right)\right\|_{0, \Omega_{0}} & \leq C \varepsilon\left(\left\|\zeta_{0}\right\|_{2, \Omega_{0}}+\left\|\zeta_{0}\right\|_{1, \Omega_{0}}\right) \\
& \leq C \frac{\varepsilon}{H_{0}}\left|\ln H_{0}\right|
\end{aligned}
$$

where we have used the following a priori estimates

$$
\left\|\zeta_{0}\right\|_{1, \Omega_{0}} \leq C\left|\ln H_{0}\right|, \quad\left\|\zeta_{0}\right\|_{2, \Omega_{0}} \leq C\left|\ln H_{0}\right| / H_{0}
$$

which can be obtained from (3.19) and from the local a priori estimates for the Green function $u_{0}$ (cf. (3.17)) by checking the governing equation for the new variable $w=\zeta_{0}-\phi u_{0}$ with the cut-off function $\phi$ introduced in (2.4).

What's left is to bound the term $\left\|\nabla \bar{\zeta}_{\varepsilon}\right\|_{0, \Omega_{0}}$. We split $\bar{\zeta}_{\varepsilon}$ into two parts $\bar{\zeta}_{\varepsilon}=\hat{\zeta}_{\varepsilon}+\tilde{\zeta}_{\varepsilon}$ by

$$
\begin{aligned}
& -\operatorname{div}\left(K_{\varepsilon} \nabla \hat{\zeta}_{\varepsilon}\right)=0 \text { in } \Omega_{0}, \\
& \left.\hat{\zeta}_{\varepsilon}\right|_{\partial \Omega_{0}}=u_{\varepsilon}-u_{0}+\varepsilon \chi_{i} \frac{\partial u_{0}}{\partial x_{i}}-\varepsilon \theta_{\varepsilon}\left(u_{0}\right),
\end{aligned}
$$

and

$$
\begin{aligned}
& -\operatorname{div}\left(K_{\varepsilon} \nabla \tilde{\zeta}_{\varepsilon}\right)=0 \text { in } \Omega_{0}, \\
& \left.\tilde{\zeta}_{\varepsilon}\right|_{\partial \Omega_{0}}=-\varepsilon \chi_{i} \frac{\partial u_{0}}{\partial x_{i}}+\varepsilon \theta_{\varepsilon}\left(u_{0}\right) .
\end{aligned}
$$


For the first part $\hat{\zeta}_{\varepsilon}$, recalling the cut-off function $\phi \in C^{1}\left(\Omega_{0}\right)$ introduced in $(2.4), \phi \equiv 0$ in $B\left(x_{0}, H_{0} / 2\right), \phi \equiv 1$ in $\Omega_{0} \backslash B\left(x_{0}, 3 H_{0} / 4\right)$ such that $|\nabla \phi| \leq \frac{C}{H_{0}}$, we have

$$
\begin{aligned}
\left\|\nabla \hat{\zeta}_{\varepsilon}\right\|_{0, \Omega_{0}} \leq & C\left\|\nabla\left(\phi\left(u_{\varepsilon}-u_{0}+\varepsilon \chi_{i} \frac{\partial u_{0}}{\partial x_{i}}-\varepsilon \theta_{\varepsilon}\left(u_{0}\right)\right)\right)\right\|_{0, \Omega_{0}} \\
\leq & C\left\|\nabla\left(u_{\varepsilon}-u_{0}+\varepsilon \chi_{i} \frac{\partial u_{0}}{\partial x_{i}}-\varepsilon \theta_{\varepsilon}\left(u_{0}\right)\right)\right\|_{0, \Omega_{0} \backslash B\left(x_{0}, H_{0} / 2\right)} \\
& +C\left\|\nabla \phi\left(u_{\varepsilon}-u_{0}+\varepsilon \chi_{i} \frac{\partial u_{0}}{\partial x_{i}}-\varepsilon \theta_{\varepsilon}\left(u_{0}\right)\right)\right\|_{0, \Omega_{0} \backslash B\left(x_{0}, H_{0} / 2\right)} \leq C \frac{\varepsilon}{H_{0}}|\ln \varepsilon|,
\end{aligned}
$$

where we have used the previous results (3.16), (3.15) and (3.17).

The second part $\tilde{\zeta}_{\varepsilon}$ can be regarded as a boundary corrector, so similarly to bound the corrector $\theta_{\varepsilon}\left(w_{0}\right)$ in Theorem 3.1 (see, e.g. [2]), we first introduce a cut-off function $\psi_{\varepsilon} \in C^{2}\left(\Omega_{0}\right), 0 \leq \psi_{\varepsilon} \leq 1$ in $\Omega_{0}, \psi_{\varepsilon}=1$ on the boundary $\partial \Omega_{0}, \psi_{\varepsilon} \equiv 0$ outside the $\varepsilon$-neighborhood $\Omega_{0}^{\varepsilon} \subset \Omega_{0}$ of the boundary $\partial \Omega_{0}$, and $\left|\nabla \psi_{\varepsilon}\right| \leq C / \varepsilon$ in $\Omega_{0}$ with $C$ independent of $\varepsilon$ and $\Omega_{0}$. It is clear that $\left|\Omega_{0}^{\varepsilon}\right| \leq \varepsilon\left|\partial \Omega_{0}\right| \leq C \varepsilon H_{0}$. Then thanks to (3.17),

$$
\begin{aligned}
\left\|\nabla \tilde{\zeta}_{\varepsilon}\right\|_{0, \Omega_{0}} & \leq C\left\|\varepsilon \nabla\left(\psi_{\varepsilon}\left(-\chi_{i} \frac{\partial u_{0}}{\partial x_{i}}+\theta_{\varepsilon}\left(u_{0}\right)\right)\right)\right\|_{0, \Omega_{0}} \\
& \leq C \sqrt{\left|\Omega_{0}^{\varepsilon}\right|}\left\|\theta_{\varepsilon}\left(u_{0}\right)-\chi_{i} \frac{\partial u_{0}}{\partial x_{i}}\right\|_{0, \infty, \Omega_{0}^{\varepsilon}}+\left\|\varepsilon \nabla\left(\theta_{\varepsilon}\left(u_{0}\right)-\chi_{i} \frac{\partial u_{0}}{\partial x_{i}}\right)\right\|_{0, \Omega_{0}^{\varepsilon}} \\
& \leq C \sqrt{\varepsilon H_{0}}\left\|u_{0}\right\|_{1, \infty, \Omega \backslash B\left(x_{0}, H_{0} / 2\right)}+C \varepsilon\left\|u_{0}\right\|_{2, \Omega \backslash B\left(x_{0}, H_{0} / 2\right)} \\
& \leq C\left(\sqrt{\frac{\varepsilon}{H_{0}}}+\frac{\varepsilon}{H_{0}}\right) .
\end{aligned}
$$

Combining (3.21), (3.25) and (3.26), we obtain (3.14), and the proof is completed.

\section{ERror estimates}

We first derive the error estimate for the numerical approximation of the multiscale finite element basis functions.

Lemma 4.1. For any $\chi \in X_{H}$, if $\left.\chi\right|_{T}=\sum_{i=1}^{3} c_{i} \bar{\psi}_{i}^{T}, T \in \mathcal{M}_{H}$, then its fully discrete counterpart $\chi_{h} \in Y_{H}$ is defined as $\left.\chi_{h}\right|_{T}=\sum_{i=1}^{3} c_{i} \bar{\psi}_{i}^{T, h}$, where $\bar{\psi}_{i}^{T, h}, i=1,2,3$, are the numerically constructed bases, and its counterpart in $V_{H}$ has been defined in (2.3) as $\hat{\chi}=\Pi_{H} \chi$. The following error estimate is valid

$$
\left\|\chi-\chi_{h}\right\|_{H} \leq C \frac{h}{\varepsilon}\|\nabla \hat{\chi}\|_{0, \Omega}
$$

Proof. Thanks to (2.1) and (2.2), for each macro element $S \supset T \in \mathcal{M}_{H}$,

$$
\left\{\begin{array}{r}
-\nabla \cdot\left(K_{\varepsilon} \nabla \chi\right)=0 \text { in } S \\
\chi=\hat{\chi} \text { on } \partial S
\end{array}\right.
$$

$\chi_{h}$ is actually the piecewise linear finite element solution of the above problem on fine grid of size $h$. Noting that $\hat{\chi} \in P_{1}(S)$, it is direct to deduce that

$$
\left\|\nabla\left(\chi-\chi_{h}\right)\right\|_{0, S} \leq C h|\chi|_{2, S} \leq C \frac{h}{\varepsilon}\|\nabla \hat{\chi}\|_{0, S} \leq C \frac{h}{\varepsilon}\|\nabla \hat{\chi}\|_{0, T},
$$


where we have used the assumption (H1) and the a priori estimate $|\chi|_{2, S} \leq(C / \varepsilon)\|\nabla \hat{\chi}\|_{0, S}$, which can be seen easily if we write down the governing equation for the auxiliary variable $w=\chi-\hat{\chi}$

$$
\left\{\begin{array}{r}
-\Delta w=\frac{1}{K_{\varepsilon}} \nabla K_{\varepsilon} \cdot \nabla \chi \text { in } S \\
w=0 \text { on } \partial S
\end{array}\right.
$$

The standard elliptic regularity yields that $|w|_{2, S} \leq(C / \varepsilon)\|\nabla \chi\|_{0, S} \leq(C / \varepsilon)\|\nabla \hat{\chi}\|_{0, S}$.

Finally, we have

$$
\left\|\chi-\chi_{h}\right\|_{H}^{2}=\sum_{T \in \mathcal{M}_{H}} \int_{T}\left|\nabla\left(\chi-\chi_{h}\right)\right|^{2} \mathrm{~d} x \leq \sum_{T \in \mathcal{M}_{H}} \int_{S}\left|\nabla\left(\chi-\chi_{h}\right)\right|^{2} \leq C\left(\frac{h}{\varepsilon}\right)^{2}\|\nabla \hat{\chi}\|_{0, \Omega}^{2}
$$

We are now going to prove Theorem 2.1. Before that, we list some previous results. The following lemma can be found in [3], Section 5.

Lemma 4.2. Under the assumptions (H1)-(H2) there exist positive constants $C$ independent of $H, \varepsilon$ such that for sufficiently small $H>0$, the following estimates are valid

$$
\begin{aligned}
& \left\|\nabla \hat{\chi}_{H}\right\|_{0, T} \leq C\left\|\nabla \chi_{H}\right\|_{0, T}, \quad\left\|\nabla \chi_{H}\right\|_{0, T} \leq C\left\|\nabla \hat{\chi}_{H}\right\|_{0, T} \\
& \left\|\nabla\left(\chi_{H}-\hat{\chi}_{H}+\varepsilon \chi_{i} \frac{\partial \hat{\chi}_{H}}{\partial x_{i}}-\varepsilon \tilde{\theta}_{\varepsilon}\left(\hat{\chi}_{H}\right)\right)\right\|_{0, T} \leq C\left(\frac{\varepsilon}{H}+H\right)\left\|\nabla \hat{\chi}_{H}\right\|_{0, T}
\end{aligned}
$$

for any $\chi_{H} \in X_{H}, \hat{\chi}_{H}=\Pi_{H} \chi_{H} \in V_{H}$, where $\tilde{\theta}_{\varepsilon}\left(\hat{\chi}_{H}\right)$ is the associated boundary corrector that satisfies, for any $T \in \mathcal{M}_{H}, S=S(T)$ the over-sampling element,

$$
-\operatorname{div}\left(K_{\varepsilon}(x) \nabla \tilde{\theta}_{\varepsilon}\left(\hat{\chi}_{H}\right)\right)=0 \quad \text { in } S,\left.\quad \tilde{\theta}_{\varepsilon}\left(\hat{\chi}_{H}\right)\right|_{\partial S}=\chi^{k}\left(x, \frac{x}{\varepsilon}\right) \frac{\partial \hat{\chi}_{H}}{\partial x_{k}} \quad \text { on } \partial S,
$$

and $\tilde{\theta}_{\varepsilon}\left(\hat{\chi}_{H}\right)$ is bounded in [7] by

$$
\left\|\nabla \tilde{\theta}_{\varepsilon}\left(\hat{\chi}_{H}\right)\right\|_{0, T} \leq C \frac{\varepsilon}{H}\left\|\nabla \hat{\chi}_{H}\right\|_{0, T}
$$

The following result is well known (cf. [7]).

Lemma 4.3. Let $N(x, y)$ be periodic in $y$ with respect to the unit square $Y$ in $\mathbf{R}^{2}$ such that $\int_{Y} N(x, y) \mathrm{d} y=0$ for any $x \in D$. Moreover, assume that $|N(x, y)|+\left|\nabla_{x} N(x, y)\right| \leq C$ for any $x \in D, y \in \mathbf{R}^{2}$. Then, for any $\xi \in H^{1}(D) \cap L^{\infty}(D)$, we have

$$
\left|\int_{D} \xi(x) N\left(x, \frac{x}{\varepsilon}\right) \mathrm{d} x\right| \leq C \varepsilon|D|^{1 / 2}\|\xi\|_{1, D}+C \varepsilon|\partial D|\|\xi\|_{0, \infty, D}
$$

The next lemma, which considered the local error between the local Green function $G_{\varepsilon}$ and its fine scale piecewise linear finite element approximation $G_{h}$, can be found in [3], Theorem 6.2.

Lemma 4.4. There exists a constant $C$ independent of $h$ and $H_{0}$ such that

$$
\left\|\nabla\left(G_{\varepsilon}-G_{h}\right)\right\|_{0, \Omega_{0} \backslash \bar{B}\left(x_{0}, H_{0} / 2\right)} \leq C \frac{h}{\varepsilon H_{0}} .
$$


Proof of Theorem 2.1. First by Strang's second lemma, we have from (1.3) and (2.5) that

$$
\begin{aligned}
\left\|\zeta_{\varepsilon}-\zeta_{H}\right\|_{H} \leq & C \inf _{\chi_{H} \in Y_{H}^{0}}\left\|\zeta_{\varepsilon}-\chi_{H}\right\|_{H} \\
& +\quad C \sup _{\chi_{H} \in Y_{H}^{0}} \frac{\left|\left(K_{\varepsilon} \nabla G_{h}, \nabla\left(\phi \hat{\chi}_{H}\right)\right)_{\Omega_{0}}-\sum_{T \in \mathcal{M}_{H}}\left(K_{\varepsilon} \nabla \zeta_{\varepsilon}, \nabla \chi_{H}\right)_{T}\right|}{\left\|\chi_{H}\right\|_{H}} .
\end{aligned}
$$

In (4.6) the error is divided into two parts "conforming error" and "nonconforming error". The "conforming error" is dominated by the interpolation error. For $\zeta_{0}$, we may define its different node interpolations: $I_{X} \zeta_{0} \in$ $X_{H}^{0}, I_{Y} \zeta_{0} \in Y_{H}^{0}$ and $I_{V} \zeta_{0} \in V_{H}^{0}$ as for each $T \in \mathcal{M}_{H},\left.I_{X} \zeta_{0}\right|_{T}=\sum_{i=1}^{3} \zeta_{0}\left(x_{i}\right) \bar{\psi}_{i}^{T}$, where $x_{i}, i=1,2,3$, are the vertices of $T$, and $\bar{\psi}_{i}^{T}, i=1,2,3$, are the corresponding over-sampling finite element bases $(c f .(2.2)) ; I_{Y} \zeta_{0}$ and $I_{V} \zeta_{0}$ are defined in the same way.

Split the interpolation error into two parts

$$
\left\|\zeta_{\varepsilon}-I_{Y} \zeta_{0}\right\|_{H} \leq\left\|\zeta_{\varepsilon}-I_{X} \zeta_{0}\right\|_{H}+\left\|I_{X} \zeta_{0}-I_{Y} \zeta_{0}\right\|_{H}
$$

The first part is also the interpolation error for the multiscale finite element space $X_{H}$, and the second part is the error due to the numerical approximation of the finite element basis functions. Thanks to Lemma 4.1, (3.17) and (3.22), we have

$$
\left\|I_{X} \zeta_{0}-I_{Y} \zeta_{0}\right\|_{H} \leq C \frac{h}{\varepsilon}\left\|\nabla\left(\Pi_{H} I_{X} \zeta_{0}\right)\right\|_{0, \Omega}=C \frac{h}{\varepsilon}\left\|\nabla I_{V} \zeta_{0}\right\|_{0, \Omega} \leq C \frac{h}{\varepsilon}\left|\ln H_{0}\right|
$$

The interpolation error in the right hand side of (4.7) can be treated under the framework of [7], Section 3.1, though some more attention should be paid on the lack of global regularity and on the precise dependence on the different length scales $\varepsilon, H, H_{0}$.

First we begin with

$$
\left\|\zeta_{\varepsilon}-I_{X} \zeta_{0}\right\|_{H}^{2}=\left\|\zeta_{\varepsilon}-I_{X} \zeta_{0}\right\|_{H, \Omega \backslash \Omega_{0}}^{2}+\left\|\zeta_{\varepsilon}-I_{X} \zeta_{0}\right\|_{H, \Omega_{0}}^{2}
$$

Denoting by $v_{H}=I_{X} \zeta_{0} \in X_{H}^{0}$ and by $\hat{v}_{H}=\Pi_{H}\left(I_{X} \zeta_{0}\right)=I_{V} \zeta_{0} \in V_{H}^{0}$, we have for any $T \in \mathcal{M}_{H} \cap\left(\Omega \backslash \Omega_{0}\right)$

$$
\begin{aligned}
\left\|\zeta_{\varepsilon}-I_{X} \zeta_{0}\right\|_{0, T} \leq\left\|\nabla\left(\zeta_{\varepsilon}-\zeta_{0}-\varepsilon \chi_{i} \frac{\partial \zeta_{0}}{\partial x_{i}}+\varepsilon \theta_{\varepsilon}\left(\zeta_{0}\right)\right)\right\|_{0, T} \\
+\left\|\nabla\left(v_{H}-\hat{v}_{H}-\varepsilon \chi_{i} \frac{\partial \hat{v}_{H}}{\partial x_{i}}+\varepsilon \tilde{\theta}_{\varepsilon}\left(\hat{v}_{H}\right)\right)\right\|_{0, T}+\left\|\nabla\left(\zeta_{0}-\hat{v}_{H}\right)\right\|_{0, T} \\
\quad+\left\|\nabla\left(\varepsilon \chi_{i} \frac{\partial}{\partial x_{i}}\left(\zeta_{0}-\hat{v}_{H}\right)\right)\right\|_{0, T}+\left\|\varepsilon \nabla \theta_{\varepsilon}\left(\zeta_{0}\right)\right\|_{0, T}+\left\|\varepsilon \nabla \tilde{\theta}_{\varepsilon}\left(\hat{v}_{H}\right)\right\|_{0, T} .
\end{aligned}
$$

Then we have

$$
\begin{aligned}
\| \zeta_{\varepsilon}- & I_{X} \zeta_{0}\left\|_{H, \Omega \backslash \Omega_{0}} \leq\right\| \nabla\left(\zeta_{\varepsilon}-\zeta_{0}-\varepsilon \chi_{i} \frac{\partial \zeta_{0}}{\partial x_{i}}+\varepsilon \theta_{\varepsilon}\left(\zeta_{0}\right)\right) \|_{0, \Omega \backslash \Omega_{0}} \\
& +\left(\sum_{T \in \Omega \backslash \Omega_{0}}\left\|\nabla\left(v_{H}-\hat{v}_{H}-\varepsilon \chi_{i} \frac{\partial \hat{v}_{H}}{\partial x_{i}}+\varepsilon \tilde{\theta}_{\varepsilon}\left(\hat{v}_{H}\right)\right)\right\|_{0, T}^{2}\right)^{1 / 2}+\left\|\nabla\left(\zeta_{0}-\hat{v}_{H}\right)\right\|_{0, \Omega \backslash \Omega_{0}} \\
& +\left\|\varepsilon \nabla \theta_{\varepsilon}\left(\zeta_{0}\right)\right\|_{0, \Omega \backslash \Omega_{0}}+\left(\sum_{T \in \Omega \backslash \Omega_{0}}\left\|\nabla\left(\varepsilon \chi_{i} \frac{\partial}{\partial x_{i}}\left(\zeta_{0}-\hat{v}_{H}\right)\right)\right\|_{0, T}^{2}\right)^{1 / 2} \\
& +\left(\sum_{T \in \Omega \backslash \Omega_{0}}\left\|\varepsilon \nabla \tilde{\theta}_{\varepsilon}\left(\hat{v}_{H}\right)\right\|_{0, T}^{2}\right)^{1 / 2} \equiv I_{1}+I_{2}+\cdots+I_{6} .
\end{aligned}
$$


The first two terms have been bounded in Theorem 3.2 and Lemma 4.2 respectively. The third term is the standard interpolation error. Due to (3.8) and (3.17), we have

$$
I_{4} \leq\left\|\varepsilon \nabla \theta_{\varepsilon}\left(\zeta_{0}\right)\right\|_{0, \Omega} \leq C(\varepsilon+\sqrt{\varepsilon}) .
$$

The fifth term can be estimated from (3.17) as

$$
I_{5} \leq C(\varepsilon+H)\left\|\zeta_{0}\right\|_{2, \Omega \backslash \Omega_{0}} \leq C(\varepsilon+H) / H_{0} .
$$

Thanks to (4.5) and (3.17),

$$
I_{6} \leq C \frac{\varepsilon}{H}\left\|\nabla \hat{v}_{H}\right\|_{0, \Omega \backslash \Omega_{0}} \leq C \frac{\varepsilon}{H}\left\|\nabla \zeta_{0}\right\|_{0, \Omega \backslash \Omega_{0}} \leq C \frac{\varepsilon}{H}\left|\ln H_{0}\right| .
$$

Combining all the six terms together, we have

$$
\left\|\zeta_{\varepsilon}-I_{X} \zeta_{0}\right\|_{H, \Omega \backslash \Omega_{0}} \leq C\left(\frac{\varepsilon}{H_{0}}|\ln \varepsilon|+\left(\frac{\varepsilon}{H}+H\right)\left|\ln H_{0}\right|+\frac{H}{H_{0}}+\sqrt{\varepsilon}\right) .
$$

For the second term on the right hand side of (4.9), we have

$$
\begin{aligned}
& \left\|\zeta_{\varepsilon}-I_{X} \zeta_{0}\right\|_{H, \Omega_{0}} \leq\left\|\nabla\left(\zeta_{\varepsilon}-\zeta_{0}-\varepsilon \chi_{i} \frac{\partial \zeta_{0}}{\partial x_{i}}+\varepsilon \bar{\theta}_{\varepsilon}\left(\zeta_{0}\right)\right)\right\|_{0, \Omega_{0}} \\
& \quad+\left(\sum_{T \in \Omega_{0}}\left\|\nabla\left(v_{H}-\hat{v}_{H}-\varepsilon \chi_{i} \frac{\partial \hat{v}_{H}}{\partial x_{i}}+\varepsilon \tilde{\theta}_{\varepsilon}\left(\hat{v}_{H}\right)\right)\right\|_{0, T}^{2}\right)^{1 / 2}+\left\|\nabla\left(\zeta_{0}-\hat{v}_{H}\right)\right\|_{0, \Omega_{0}} \\
& \quad+\left\|\varepsilon \nabla \bar{\theta}_{\varepsilon}\left(\zeta_{0}\right)\right\|_{0, \Omega_{0}}+\left(\sum_{T \in \Omega_{0}}\left\|\nabla\left(\varepsilon \chi_{i} \frac{\partial}{\partial x_{i}}\left(\zeta_{0}-\hat{v}_{H}\right)\right)\right\|_{0, T}^{2}\right)^{1 / 2} \\
& \left.\quad+\left(\sum_{T \in \Omega_{0}} \| \varepsilon \nabla \tilde{\theta}_{\varepsilon}\left(\hat{v}_{H}\right)\right) \|_{0, T}^{2}\right)^{1 / 2} \equiv \hat{I}_{1}+\hat{I}_{2}+\cdots+\hat{I}_{6} .
\end{aligned}
$$

The first term has been bounded in Theorem 3.2. Thanks to Lemma 4.2 and (3.22), we have

$$
\hat{I}_{2} \leq C\left(\frac{\varepsilon}{H}+H\right)\left\|\nabla \hat{v}_{H}\right\|_{0, \Omega_{0}} \leq C\left(\frac{\varepsilon}{H}+H\right)\left\|\nabla \zeta_{0}\right\|_{0, \Omega_{0}} \leq C\left(\frac{\varepsilon}{H}+H\right)\left|\ln H_{0}\right| .
$$

For the third and fifth terms, we have by (3.22)

$$
\hat{I}_{3}+\hat{I}_{5} \leq C(H+\varepsilon)\left\|\zeta_{0}\right\|_{2, \Omega_{0}} \leq C(H+\varepsilon)\left|\ln H_{0}\right| / H_{0}
$$

The fourth term can be estimated in the same way as in (4.11). Thanks to (3.8) and (3.22),

$$
\begin{aligned}
\hat{I}_{4} & \leq\left\|\varepsilon \nabla \bar{\theta}_{\varepsilon}\left(\zeta_{0}\right)\right\|_{0, \Omega_{0}} \leq C\left(\varepsilon\left|\zeta_{0}\right|_{2, \Omega_{0}^{\varepsilon}}+\sqrt{\varepsilon H_{0}}\left|\zeta_{0}\right|_{1, \infty, \Omega_{0}^{\varepsilon}}\right) \\
& \leq C\left(\frac{\varepsilon}{H}_{0}\left|\ln H_{0}\right|+\sqrt{\frac{\varepsilon}{H_{0}}}\right)
\end{aligned}
$$

where $\Omega_{0}^{\varepsilon} \subset \Omega_{0}$ is the $\varepsilon$-neighborhood of $\partial \Omega_{0}$ and (3.17) has been used to obtain $\left|\zeta_{0}\right|_{1, \infty, \Omega_{0}^{\varepsilon}}=\left|u_{0}-G_{0}\right|_{1, \infty, \Omega_{0}^{\varepsilon}} \leq$ $\mathrm{C} / \mathrm{H}_{0}$.

Due to (3.22), the sixth term can be bounded by

$$
\hat{I}_{6} \leq C \frac{\varepsilon}{H}\left\|\nabla \hat{v}_{H}\right\|_{0, \Omega_{0}} \leq C \frac{\varepsilon}{H}\left\|\nabla \zeta_{0}\right\|_{0, \Omega_{0}} \leq C \frac{\varepsilon}{H}\left|\ln H_{0}\right| .
$$


Then we obtain

$$
\left\|\zeta_{\varepsilon}-I_{X} \zeta_{0}\right\|_{H, \Omega_{0}} \leq C\left(\frac{\varepsilon}{H_{0}}|\ln \varepsilon|+\sqrt{\frac{\varepsilon}{H_{0}}}+\left(\frac{\varepsilon}{H}+\frac{\varepsilon+H}{H_{0}}\right)\left|\ln H_{0}\right|\right)
$$

Hence, combining (4.8), (4.9), (4.13) and (4.16), the "conforming error" can be bounded by

$$
\inf _{\chi_{H} \in Y_{H}^{0}}\left\|\zeta_{\varepsilon}-\chi_{H}\right\|_{H} \leq C\left(\frac{\varepsilon}{H_{0}}|\ln \varepsilon|+\sqrt{\frac{\varepsilon}{H_{0}}}+\left(\frac{\varepsilon}{H}+\frac{\varepsilon+H}{H_{0}}+\frac{h}{\varepsilon}\right)\left|\ln H_{0}\right|\right) .
$$

We now turn to bound the "nonconforming error" in (4.6). Due to (2.4), for $\chi_{H} \in Y_{H}^{0}$,

$$
\begin{gathered}
\left(K_{\varepsilon} \nabla G_{h}, \nabla\left(\phi \hat{\chi}_{H}\right)\right)_{\Omega_{0}}-\sum_{T \in \mathcal{M}_{H}}\left(K_{\varepsilon} \nabla \zeta_{\varepsilon}, \nabla \chi_{H}\right)_{T}=\left(K_{\varepsilon} \nabla G_{h}-K_{\varepsilon} \nabla G_{\varepsilon}, \nabla\left(\phi \hat{\chi}_{H}\right)\right)_{\Omega_{0}} \\
+\sum_{T \in \mathcal{M}_{H}}\left(K_{\varepsilon} \nabla \zeta_{\varepsilon}, \nabla\left(\bar{\chi}_{H}-\chi_{H}\right)\right)_{T}+\sum_{T \in \mathcal{M}_{H}}\left(K_{\varepsilon} \nabla \zeta_{\varepsilon}, \nabla\left(\hat{\chi}_{H}-\bar{\chi}_{H}\right)\right)_{T}
\end{gathered}
$$

where $\bar{\chi}_{H} \in X_{H}^{0}$ is the counterpart of $\chi_{H} \in Y_{H}^{0}$. To bound the first term on the right hand side, recalling the definition of the cut-off function $\phi$ in (2.4), we have $|\nabla \phi| \leq C H_{0}^{-1}$, so for any $w_{H} \in V_{H}$,

$$
\begin{aligned}
\left\|\nabla\left(\phi w_{H}\right)\right\|_{0, \Omega_{0}} & \leq \frac{C}{H_{0}}\left|B\left(x_{0}, 3 H_{0} / 4\right) \backslash B\left(x_{0}, H_{0} / 2\right)\right|\left\|w_{H}\right\|_{0, \infty, \Omega_{0}}+\left\|\nabla w_{H}\right\|_{0, \Omega_{0}} \\
& \leq C|\ln H|^{1 / 2}\left\|\nabla w_{H}\right\|_{0, \Omega},
\end{aligned}
$$

where we have used the well known 2-d discrete interpolate inequality

$$
\left\|w_{H}\right\|_{0, \infty, \Omega} \leq C|\ln H|^{1 / 2}\left\|\nabla w_{H}\right\|_{0, \Omega} \text { for } w_{H} \in V_{H}
$$

Due to Lemma 4.4, we obtain

$$
\left(K_{\varepsilon} \nabla G_{h}-K_{\varepsilon} \nabla G_{\varepsilon}, \nabla\left(\phi \hat{\chi}_{H}\right)\right)_{\Omega_{0}} \leq C \frac{h}{\varepsilon H_{0}}|\ln H|^{1 / 2}\left\|\nabla \hat{\chi}_{H}\right\|_{0, \Omega} .
$$

The second term on the right hand side of (4.18) is the error due to the numerical approximation of the multiscale finite element basis. Thanks to Lemma 4.1,

$$
\sum_{T \in \mathcal{M}_{H}}\left(K_{\varepsilon} \nabla \zeta_{\varepsilon}, \nabla\left(\bar{\chi}_{H}-\chi_{H}\right)\right)_{T} \leq C\left\|\nabla \zeta_{\varepsilon}\right\|_{0, \Omega}\left\|\nabla\left(\bar{\chi}_{H}-\chi_{H}\right)\right\|_{H} \leq C \frac{h}{\varepsilon}\left|\ln H_{0}\right|\left\|\nabla \hat{\chi}_{H}\right\|_{0, \Omega}
$$

where we have used the a priori estimate $\left\|\nabla \zeta_{\varepsilon}\right\|_{0, \Omega} \leq C\left|\ln H_{0}\right|$, which can be obtain by choosing $v=\zeta_{\varepsilon}$ in $(2.4)$.

The third term on the right hand side of (4.18) can be estimated by the similar way as in [7], Section 3.1. Noting that $\hat{\chi}_{H}$ is piecewise linear, for any $T \in \mathcal{M}_{H}$, we have

$$
\begin{aligned}
\int_{T} K_{\varepsilon} \nabla \zeta_{\varepsilon} \nabla & \left(\bar{\chi}_{H}-\hat{\chi}_{H}\right)=\int_{T} K_{\varepsilon} \nabla \zeta_{\varepsilon} \nabla\left(\bar{\chi}_{H}-\hat{\chi}_{H}+\varepsilon \chi_{i}\left(x, \frac{x}{\varepsilon}\right) \frac{\partial \hat{\chi}_{H}}{\partial x_{i}}\right) \mathrm{d} x \\
& -\int_{T} K_{\varepsilon} \frac{\partial \zeta_{\varepsilon}}{\partial x_{j}} \varepsilon \frac{\partial}{\partial x_{j}} \chi_{i}(x, y) \frac{\partial \hat{\chi}_{H}}{\partial x_{i}} \mathrm{~d} x-\int_{T} K_{\varepsilon} \frac{\partial \zeta_{\varepsilon}}{\partial x_{j}} \frac{\partial}{\partial y_{j}} \chi_{i}(x, y) \frac{\partial \hat{\chi}_{H}}{\partial x_{i}} \mathrm{~d} x . \\
& \equiv T_{1}+T_{2}+T_{3} .
\end{aligned}
$$


We have from Lemma 4.2,

$$
\begin{aligned}
\sum_{T \in \mathcal{M}_{H}}\left|T_{1}\right|+\left|T_{2}\right| & \leq \sum_{T \in \mathcal{M}_{H}} C\left(\varepsilon+\frac{\varepsilon}{H}+H\right)\left\|\nabla \zeta_{\varepsilon}\right\|_{0, T}\left\|\nabla \hat{\chi}_{H}\right\|_{0, T} \\
& \leq C\left(\varepsilon+\frac{\varepsilon}{H}+H\right)\left\|\nabla \zeta_{\varepsilon}\right\|_{0, \Omega}\left\|\nabla \hat{\chi}_{H}\right\|_{0, \Omega} .
\end{aligned}
$$

Denoting by $\zeta_{1}^{\varepsilon}=\zeta_{0}-\varepsilon \chi_{j} \frac{\partial \zeta_{0}}{\partial x_{j}}$, for any $T \in \mathcal{M}_{H} \cap \Omega \backslash \Omega_{0}$,

$$
\begin{aligned}
T_{3}= & -\int_{T} K_{\varepsilon} \frac{\partial}{\partial x_{i}}\left(\zeta_{\varepsilon}-\zeta_{1}^{\varepsilon}-\varepsilon \theta_{\varepsilon}\left(\zeta_{0}\right)\right) \frac{\partial}{\partial y_{i}} \chi_{p}(x, y) \frac{\partial \hat{\chi}_{H}}{\partial x_{p}} \mathrm{~d} x \\
& -\int_{T}\left(K_{\varepsilon} \frac{\partial \zeta_{1}^{\varepsilon}}{\partial x_{i}}-k_{i j}^{*} \frac{\partial \zeta_{0}}{\partial x_{j}}\right) \frac{\partial}{\partial y_{i}} \chi_{p}(x, y) \frac{\partial \hat{\chi}_{H}}{\partial x_{p}} \mathrm{~d} x-\int_{T} k_{i j}^{*} \frac{\partial \zeta_{0}}{\partial x_{j}} \frac{\partial}{\partial y_{i}} \chi_{p}(x, y) \frac{\partial \hat{\chi}_{H}}{\partial x_{p}} \mathrm{~d} x \\
& -\int_{T} \varepsilon K_{\varepsilon} \frac{\partial \theta_{\varepsilon}\left(\zeta_{0}\right)}{\partial x_{i}} \frac{\partial}{\partial y_{i}} \chi_{p}(x, y) \frac{\partial \hat{\chi}_{H}}{\partial x_{p}} \mathrm{~d} x \equiv T_{31}+T_{32}+T_{33}+T_{34} .
\end{aligned}
$$

For $T \in \mathcal{M}_{H} \cap \Omega_{0}, \theta_{\varepsilon}\left(\zeta_{0}\right)$ should be replaced by $\bar{\theta}_{\varepsilon}\left(\zeta_{0}\right)$ in the above formula. The terms $T_{31}$ and $T_{34}$ can be bounded by using Theorems 3.2 and 3.1. The term $T_{33}$ can be estimated by using Lemma 4.3 . Term $T_{32}$ can be treated by the standard argument (cf. [12], Sect. 1.3, (1.48)). Hence we have

$$
\left|T_{32}\right|+\left|T_{33}\right| \leq C \varepsilon\left(\left|\zeta_{0}\right|_{2, T}+\left|\zeta_{0}\right|_{1, \infty, T}\right)\left\|\nabla \hat{\chi}_{H}\right\|_{0, T}
$$

and by using (4.12) and (4.15),

$$
\begin{aligned}
\left|\sum_{T \in \mathcal{M}_{H}} T_{3}\right|= & \left|\sum_{T \in \Omega \backslash \Omega_{0}} T_{3}+\sum_{T \in \Omega_{0}} T_{3}\right| \leq C\left(\frac{\varepsilon}{H_{0}}|\ln \varepsilon|+\sqrt{\varepsilon}+\frac{\varepsilon}{H_{0}}+\frac{\varepsilon}{H_{0} H}\right)\left\|\nabla \hat{\chi}_{H}\right\|_{0, \Omega \backslash \Omega_{0}} \\
& +C\left(\frac{\varepsilon}{H_{0}}|\ln \varepsilon|+\sqrt{\frac{\varepsilon}{H_{0}}}+\frac{\varepsilon}{H_{0}}\left|\ln H_{0}\right|+\frac{\varepsilon}{H}\left|\ln H_{0}\right|\right)\left\|\nabla \hat{\chi}_{H}\right\|_{0, \Omega_{0} .}
\end{aligned}
$$

Thanks to Lemma 4.1 and and the stability result (4.3) of Lemma 4.2 , we can deduce that for $h \ll \varepsilon \ll H \ll 1$,

$$
\left\|\nabla \hat{\chi}_{H}\right\|_{0, \Omega} \leq C\left\|_{\chi_{H}}\right\|_{H}, \forall \chi_{H} \in Y_{H}^{0}
$$

So the "nonconforming error" can be bounded by

$$
\begin{gathered}
\sup _{\chi_{H} \in Y_{H}^{0}} \frac{\left|\left(K_{\varepsilon} \nabla G_{h}, \nabla\left(\phi \hat{\chi}_{H}\right)\right)_{\Omega_{0}}-\sum_{T \in \mathcal{M}_{H}}\left(K_{\varepsilon} \nabla \zeta_{\varepsilon}, \nabla \chi_{H}\right)_{T}\right|}{\left\|\chi_{H}\right\|_{H}} \leq C\left(\frac{\varepsilon}{H_{0}}|\ln \varepsilon|+\sqrt{\frac{\varepsilon}{H_{0}}}\right. \\
\left.+\frac{\varepsilon}{H_{0} H}+\left(\varepsilon+H+\frac{\varepsilon}{H}+\frac{\varepsilon}{H_{0}}+\frac{h}{\varepsilon}\right)\left|\ln H_{0}\right|\right) .
\end{gathered}
$$

Finally combining the conforming error (4.17) and nonconforming error (4.25) together, we complete the proof of Theorem 2.1.

Acknowledgements. The authors wish to thank Prof. Z.M. Chen for many inspiring discussions on this topic. They'd also like to thank the referees for their valuable comments and suggestions. This work is supported in part by NSF of China under the grant 10471102 and the national basic research program under the grant 2005CB321704. 


\section{REFERENCES}

[1] I. Babuska, G. Caloz and J. Osborn, Special finite element methods for a class of second order elliptic problems with rough coefficients. SIAM J. Numer. Anal. 31 (1994) 945-981.

[2] Z. Chen and T.Y. Hou, A mixed multiscale finite element method for elliptic problem with oscillating coefficients. Math. Comp. 72 (2003) 541-576.

[3] Z. Chen and X. Yue, Numerical homogenization of well singularities in the flow transport through heterogeneous porous media. Multiscale Model. Simul. 1 (2003) 260-303.

[4] L.J. Durlofsky, Numerical-calculation of equivalent grid block permeability tensors for heterogeous porous media. Water Resour. Res. 27 (1991) 699-708.

[5] L.J. Durlofsky, W.J. Milliken and A. Bernath, Scale up in the Near-Well Region, SPE 51940, in Proceedings of the 15th SPE Symposium on Reservoir Simulation, Houston, February (1999) 14-17.

[6] W. E and B. Engquist, The heterogeneous multiscale methods. Commun. Math. Sci. 1 (2003) 87-132.

[7] Y.R. Efendiev, T.Y. Hou and X.H. Wu, The convergence of non-conforming multiscale finite element methods. SIAM J. Numer. Anal. 37 (2000) 888-910.

[8] A. Gloria, A direct approach to numerical homogenization in finite elasticity. Netw. Heterog. Media 1 (2006) 109-141.

[9] A. Gloria, A analytical framework for the numerical homogenization of monotone elliptic operators and quasiconvex energies. Multiscale Model. Simul. 5 (2006) 996-1043.

[10] T.Y. Hou and X.H. Wu, A multiscale finite element method for elliptic problems in composite materials and porous media. $J$. Comput. Phys. 134 (1997) 169-189.

[11] T.Y. Hou, X.H. Wu and Z. Cai, Convergence of a multiscale finite element method for elliptic problems with rapidly oscillating coefficients. Math. Comp. 68 (1999) 913-943.

[12] V.V. Jikov, S.M. Kozlov and O.A. Oleinik, Homogenization of Differential Operators and Integral Functionals. Springer, Berlin (1994).

[13] O. Mascarenhas and L.J. Durlofsky, Scale up in the vicinity of horizontal wells, in Proceedings of the 20th Annual International Energy Agency Workshop and Symposium, Paris, September (1999) 22-24.

[14] A.M. Matache, I. Babuska and C. Schwab, Generalized p-FEM in homogenization. Numer. Math. 86 (2000) 319-375.

[15] D.W. Peaceman, Interpretation of well-block pressures in numerical reservoir simulations. Soc. Pet. Eng. J. 18 (1978) $183-194$.

[16] X.H. Wen and J.J. Gomez-Hernandez, Upscaling hydraulic conductivities in heterogeneous media: an overview. J. Hydrol. 183 (1996) ix-xxxii. 